\title{
Création en nature avec les Amis de la montagne : vers un nécessaire retour aux sources
}

\section{Samuel Montigné}

\section{(2) OpenEdition}

\section{Journals}

Édition électronique

URL : http://journals.openedition.org/ere/2562

DOI : $10.4000 /$ ere.2562

ISSN : 2561-2271

Éditeur

Centr'ERE

Référence électronique

Samuel Montigné, "Création en nature avec les Amis de la montagne : vers un nécessaire retour aux sources », Éducation relative à l'environnement [En ligne], Volume 14 - 1 | 2017, mis en ligne le 15 septembre 2017, consulté le 21 février 2020. URL : http://journals.openedition.org/ere/2562 ; DOI : 10.4000/ere.2562

Ce document a été généré automatiquement le 21 février 2020. 


\title{
Création en nature avec les Amis de la montagne : vers un nécessaire retour aux sources
}

\author{
Samuel Montigné
}

1 À travers les époques, l'art a permis à l'humain de transcrire dans un langage symbolique son rapport conscient et inconscient à l'environnement et à l'Autre.

2 Au tournant des années 1970, alors que l'Occident commence à prendre réellement conscience des problématiques environnementales, de nombreux artistes ressentent la nécessité de s'extirper d'un monde de l'art de plus en plus conceptuel, de sortir des galeries pour travailler directement dans la nature. Cette quête de sens nécessite un retour aux sources même de la création. Au début, le mouvement Land art présente des œuvres gigantesques qui modifient durablement le paysage et qui expriment la maitrise de l'humain sur la nature. Rapidement cependant, se développe un courant plus écologiquement responsable. L'art dans la nature, par exemple, s'intéresse plutôt à des œuvres éphémères réalisées in situ, à partir des éléments présents sur place et ayant pour seule permanence le cliché photographique (Kastner, 2005, p.12). Des artistes comme Nils Udo ou Andy Goldsworthy placent en effet la nature au cœur de leur processus créatif et proposent une forme d'art plus accessible pour le public qui tente de relier l'art et la vie, tout en prônant une forme d'écologie de l'art. Au Québec, cet élan artistique a trouvé un écho durable auprès de plusieurs artistes et a favorisé l'émergence de symposiums associant art et nature ${ }^{1}$.

3 Parallèlement à ce mouvement artistique plus naturaliste, les années 1970 ont également vu l'émergence du mouvement écologiste et les débuts du champ de l'éducation relative à l'environnement (ERE). Après bientôt cinquante ans de travail dans les milieux formels et non formels, on observe toutefois que les acteurs de l'ERE sont encore et toujours confrontés à certaines limites dans l'atteinte de leurs objectifs. En effet, malgré la confirmation scientifique de l'impact des activités humaines sur l'état de l'environnement, il peut paraître surprenant que des populations de plus en 
plus informées fassent toujours preuve d'une incapacité à modifier de manière significative leurs comportements et leurs modes de vie.

4 En tant que pédagogue et praticien de l'ERE, il est nécessaire que nous nous questionnions sur cette impasse. Dominique Cottereau attire notre attention sur le fait «que la majorité des travaux [dans le domaine de l'environnement] s'orientent vers une compréhension objective $d u$ fonctionnement des systèmes terrestres [...] mais laissent en marge la source profonde qui nous relie à la terre: l'imaginaire " (Cottereau, 2001, p. 93). Se pourrait-il que nous ayons sous-estimé l'importance de la dimension affective de notre rapport à l'environnement? Se pourrait-il que cette dimension sensible et subjective soit une clé oubliée pouvant opérer une véritable transformation du rapport au monde et de l'agir collectif ?

Dans un univers social où la relation au monde passe de plus en plus par une interface numérique - laquelle contribue à aggraver la fracture entre l'humain et la nature nous ressentons plus que jamais le besoin d'un certain retour aux sources: le rétablissement d'un contact avec le milieu naturel par des approches sensibles qui favorisent une reconnexion à soi, à la nature et aux autres.

6 La pratique de l'art nature ${ }^{2}$, qui favorise le développement de l'imaginaire et de la créativité et qui «se concentre sur la relation, le dialogue avec le lieu comme moteur au processus créatif » (Loncol Daigneault, 2011, p. 87), pourrait enrichir le répertoire de stratégies de l'ERE.

7 Depuis plus de 30 ans, l'organisme Les amis de la montagne est activement engagé pour la protection et la mise en valeur du mont Royal tout en favorisant l'ERE et l'engagement collectif. Il propose à des publics variés des programmes éducatifs favorisant une relation sensible à la montagne dans le but de renforcer le sentiment d'appartenance à la nature tout en développant un agir individuel et collectif plus respectueux de la montagne et de ses composantes ${ }^{3}$.

Depuis quelques années, nous avons développé au sein de cet organisme un nouveau champ d'activités de créations artistiques en milieu naturel. Nous en présenterons ici deux exemples et montrerons en quoi ceux-ci contribuent aux visées de l'ERE tout en proposant des pistes d'interventions novatrices.

\section{Le mont Royal, un formidable espace de nature, d'histoire et de culture au cœur de la ville}

9 Situé au cœur de l'île de Montréal, le mont Royal est une colline d'origine magmatique qui constitue depuis toujours un repère visuel et identitaire pour les Montréalais. Chaque année, plus de quatre millions de touristes et de métropolitains fréquentent ce lieu incontournable.

La «montagne » comme l'appellent affectueusement les Montréalais, couvre un vaste territoire de $10 \mathrm{~km}$ carré sur lequel ont été implantés au fil du temps des institutions religieuses, des cimetières, des universités. C'est aussi un grand parc avec un espace public de nature préservée où les Montréalais aiment pratiquer différents loisirs et se ressourcer au contact de la nature.

11 Dès la création du Parc du Mont-Royal, en 1876, son concepteur, l'architecte paysagiste Frederick Law Olmsted, était convaincu de la fonction thérapeutique de la nature pour 
l'humain. Il a dessiné les plans visionnaires de ce parc en considérant que celui-ci "devrait avoir la cohérence et la force véritable d'une œuvre d'art ». Il a exploité le plein potentiel du « génie du lieu » et mis en valeur «le charme poétique des paysages naturels » qui, dit-il, « nous transforme sans que nous sachions pourquoi. Il nous aide à devenir poètes » (Chartier, 2008, p. 28-33).

L'art est également bien présent sur le mont Royal avec, notamment, les œuvres du Symposium international de sculptures de 1964, où pendant trois mois, onze artistes provenant de neuf pays différents ont façonné des sculptures en pierre et en métal en lien direct avec les éléments du site et le public. Ces œuvres, ainsi que plusieurs autres créées par la suite dans la nature, forment une extraordinaire collection d'art public.

C'est dans le cadre de cet environnement riche de nature, de culture et d'histoire, que Les amis de la montagne $e^{4}$ proposent au public de tous âges une randonnée art nature qui utilise le médium artistique comme vecteur pour créer un lien avec l'environnement. Cette randonnée invite les participants à deux activités lors du parcours :

1) dessin/peinture à partir de l'observation du paysage et des éléments naturels ;

2) création art nature à partir des éléments in situ.

\section{Des œuvres picturales pour renforcer le lien à l'environnement}

16 Avant la randonnée, nous prêtons à chaque participant un petit sac contenant le minimum de matériel pour dessiner : un crayon affûté, un pinceau avec réserve d'eau, une palette avec 8 couleurs d'aquarelle et un carnet de dessin. La simplicité du matériel facilite la logistique de l'activité et simplifie le processus de création pour permettre aux participants de se concentrer sur le rapport avec le milieu naturel.

La randonnée débute par une courte visite de la collection d'œuvres d'art public présentes en extérieur sur la montagne. Puis, les participants sont invités à faire un dessin. Ils ont droit à une petite démonstration technique pour l'utilisation du crayon et des couleurs. Il est également essentiel de donner quelques consignes sur le dessin d'observation, afin de permettre aux participants de surmonter l'obstacle de l'incompétence technique : «Je ne sais pas dessiner!» Nous leur rappelons le principe que du moment que nous savons écrire, nous avons tous la capacité de dessiner. En fait, « il n'est guère difficile de dessiner. Le problème est d'apprendre à voir » (Edwards, 1979, p. 4). Réussir à bien voir les choses implique une modification de nos modes de perceptions habituels (Tableau 1, colonne de gauche). 
Tableau 1 : Comparaison des caractéristiques des modes gauche et droit du cerveau (d'après Edwards, 1979, p. 4)

\begin{tabular}{|c|c|}
\hline Modes de perception de type " cerveau gauche " & Modes de perception de type " cerveau droit" \\
\hline $\begin{array}{l}\text { Verbal: } \\
\text { Utilise des mots pour désigner, décrire, définir. }\end{array}$ & $\begin{array}{l}\text { Non verbal: } \\
\text { Conscient des choses, mais correspondance minimale avec les } \\
\text { mots. }\end{array}$ \\
\hline $\begin{array}{l}\text { Analytique: } \\
\text { Considère les choses petit à petit et une partie après l'autre. }\end{array}$ & $\begin{array}{l}\text { Synthétique : } \\
\text { Met les choses ensemble pour former un tout. }\end{array}$ \\
\hline $\begin{array}{l}\text { Symbolique: } \\
\text { Utilise les symboles pour désigner les choses. }\end{array}$ & $\begin{array}{l}\text { Concret : } \\
\text { Se réfère aux choses telles qu'elles sont au moment présent. }\end{array}$ \\
\hline $\begin{array}{l}\text { Abstrait: } \\
\text { Relève une petite partie de l'information et l'utilise pour } \\
\text { représenter la chose dans son ensemble. }\end{array}$ & $\begin{array}{l}\text { Analogique: } \\
\text { Perçoit les ressemblances entre les choses; comprend les } \\
\text { relations métaphoriques. }\end{array}$ \\
\hline $\begin{array}{l}\text { Temporel: } \\
\text { Conscient du temps, envisage les choses dans leur ordre de } \\
\text { succession. }\end{array}$ & $\begin{array}{l}\text { Intemporel : } \\
\text { N'a pas la notion du temps. }\end{array}$ \\
\hline $\begin{array}{l}\text { Rationnel : } \\
\text { Tire des conclusions fondées sur la raison et sur des faits. }\end{array}$ & $\begin{array}{l}\text { Irrationnel: } \\
\text { N'exige pas de justifications par la raison ou par les faits; } \\
\text { désireux de surseoir aux jugements. }\end{array}$ \\
\hline $\begin{array}{l}\text { Digital: } \\
\text { Utilise des nombres par exemple pour compter. }\end{array}$ & $\begin{array}{l}\text { Spatial : } \\
\text { Perçoit les relations entre les choses et les rapports qui unissent } \\
\text { les parties d'un tout. }\end{array}$ \\
\hline $\begin{array}{l}\text { Logique : } \\
\text { Tire des conclusions conformément à la logique. }\end{array}$ & $\begin{array}{l}\text { Intuitif: } \\
\text { Appréhende instantanément la réalité souvent basée sur des } \\
\text { données incomplètes, des intuitions, des sentiments, ou des } \\
\text { images. }\end{array}$ \\
\hline $\begin{array}{l}\text { Linéaire: } \\
\text { Pense sous forme d'idées suivies, menant souvent à des } \\
\text { conclusions convergentes. }\end{array}$ & $\begin{array}{l}\text { Global : } \\
\text { Perçoit une suite de choses dans leur ensemble menant souvent à } \\
\text { des conclusions divergentes. }\end{array}$ \\
\hline
\end{tabular}

Des études plus récentes sur le cerveau viennent démystifier la séparation physique des modes de perception associés à un hémisphère cérébral en particulier (Vidal et BenoitBrowaeys, 2005). Cependant, on peut observer que notre rapport au monde, renforcé par certaines orientations éducatives (Pineau, 1977, p. 1), s'appuie généralement sur les modes de perception associés au « cerveau gauche » qui font plutôt appel au rationnel, à l'analyse et à la logique. Nous exploitons beaucoup moins les capacités de notre cerveau qui font appel à l'intuitif ou bien à une vision globale du monde. Le dessin d'observation, lui, implique et favorise une vision synthétique, non verbale. On suggère donc aux participants de s'efforcer à dessiner des lignes, des rapports d'espace, des éléments tels qu'ils apparaissent, sans les nommer ou les analyser.

19 L'éducateur désigne un espace pour réaliser cet exercice d'observation et pour dessiner. Il peut s'agir d'un point de vue ou d'un milieu plus fermé comme un coin de forêt. Chaque participant choisit un endroit confortable pour s'installer et fixe un sujet à dessiner. Pendant l'exercice, l'éducateur assure la gestion du temps et le support logistique, mais sa présence doit être relativement effacée. Les consignes et les conseils pour améliorer le travail d'observation seront prodigués individuellement par l'éducateur et de façon discrète pour interférer le moins possible dans la relation qui se met en place entre le participant et le milieu.

20 Généralement, un silence rempli par les sons subtils de la nature s'installe. Cet environnement sonore paisible induit un niveau plus élevé de concentration avec un changement de mode de perception de l'environnement, qui «suscite une légère modification de l'état de conscience avec des sensations auxquelles font référence de nombreux artistes : une sensation d'intemporalité et de communion [...] un sentiment de confiance et l'absence d'anxiété, une impression profonde pour les lignes et les surfaces, et pour des formes qui restent sans dénomination " (Edwards, 1979, p. 4). Cet état mental spécifique a été identifié par le psychologue Mihaly Csikszentmihalyi, qui l'a nommé « Flow» (Terstegge, 2013, p. 27). 
21 Dans cet état, notre rapport au temps et à l'espace se modifie. Bien que nous représentions seulement une petite partie de l'environnement sur notre carnet, c'est plus fondamentalement une sensation d'immersion dans cet espace ou d'appartenance à l'unité globale du lieu qui s'installe. Le temps est celui d'un présent que l'on vit pleinement, qui apparaît plus tangible, apaisant. Dans cet état semi-méditatif en lien avec la nature où tout notre être et tous nos sens sont ouverts, un certain sentiment d'exister apparaît, un sentiment d'apaisement, de vie. «C'est pour voir vraiment, pour voir toujours plus en profondeur et de là, pour être pleinement conscient et vivre totalement, que je dessine ce que je vois » (Frederick Frank in Edwards, 1979, p. 4).

À la vision analytique des choses se substitue celle plus synthétique, plus globale, qui est notamment capable de percevoir la beauté dans les formes de vie en présence. La mémoire imprime les formes observées ainsi que nos propres sensations.

La mise en relation à l'environnement à travers le dessin/la peinture implique donc un mode de perception particulier qui peut susciter un sentiment de bien-être et favoriser un rétablissement plus authentique du rapport à soi et aux choses. Chaque dessin, très différent d'une personne à l'autre, tend à traduire en image l'expression d'une double réalité : celles des formes observées et celle, profondément subjective, de celui qui l'a créée. Le dessin reproduit ainsi l'objet observé à travers tout le filtre de la sensibilité et du registre émotif de celui qui le produit.

24 À la fin de l'exercice, les participants qui le souhaitent peuvent présenter leur création aux autres et rendre compte de leur expérience. Bien que le processus du dessin soit de nature à favoriser une relation positive à soi et à l'environnement, il n'en demeure pas moins que les résultats observés d'après des critères purement esthétiques sont très variables et diffèrent en fonction des habilités de chacun. Il faut donc prendre les précautions nécessaires pour éviter que le jugement des pairs soit préjudiciable et altère la confiance en soi.

\section{Des créations art nature pour se reconnecter à soi, à la nature et aux autres}

25 La randonnée se poursuit. Le groupe se déplace jusqu'à un autre milieu choisi et adapté à la création art nature : un espace naturel plutôt fermé, comme un coin de forêt, présentant une diversité d'éléments naturels (roches, bois, feuillages) et permettant les déplacements libres. Il importe de s'assurer de limiter l'impact du groupe sur le milieu naturel d'autant que l'on se trouve sur une montagne, un milieu déjà fragilisé pas une grande fréquentation. Les sacs, vestes et accessoires sont déposés dans un coin pour faciliter au mieux la mobilité du corps.

Pour débuter l'atelier, l'éducateur présente au groupe quelques œuvres créées par des artistes issus du mouvement art nature. Puis, les participants sont invités à choisir dans l'espace un élément significatif ou un lieu qui leur servira d'ancrage pour amorcer leur travail de création. En général, le travail individuel est privilégié afin d'éviter le recours au langage et à la négociation, mais il est parfois réalisé en petits groupes, voire collectivement.

27 À travers le processus dynamique de création d'art nature, les participants traversent plusieurs phases qui font chacune appel à des modes de perception et d'intelligence bien différents. 


\section{Phase d'ouverture}

$\mathrm{Au}$ début de l'exercice, les participants semblent plutôt observateurs, tâtonnent timidement la matière et recherchent un point de départ. Le travail de l'éducateur consiste parfois à guider certains participants vers ce point d'ancrage essentiel à l'amorce du processus de création. Par la suite, sa présence se fait la plus discrète possible pour favoriser l'établissement d'une relation entre le participant et le milieu. À ce moment, les participants vivent une phase d'ouverture qui implique une écoute du milieu, de sa réalité physique, de ses composantes, de son potentiel esthétique et qui implique en même temps une écoute de soi, une écoute de ses sensations, de ses intérêts et de tout son potentiel créatif.

\section{Phase d'action productive}

lui, fait appel à un sens de la créativité beaucoup plus intuitif et universel. Bien que peu de consignes soient données par l'éducateur sur la forme des œuvres à produire, on observe généralement l'apparition de formes organiques plutôt abstraites et très rarement figuratives. Ces formes, récurrentes d'un groupe à l'autre - cercles, courbes, mandalas, nids ou abris -, semblent traduire la résonance du participant avec le milieu dans lequel il se trouve, mais surtout avec sa nature profonde d'être vivant. Roche, bois, feuille, terre, chaque élément possède une matérialité, une sensation tactile et une présence visuelle qui lui est propre. Le choix des matériaux semble être, pour chaque participant, le résultat de ce dialogue matériel et symbolique avec le milieu. La pratique de l'art nature ouvre de riches perspectives éducatives :

En ayant simplement inclus des formes éducatives existantes mais négligées des axes prioritaires - l'imaginaire créateur, le sens du jeu, l'écoute sensible des éléments -, on a ouvert une porte sur l'inconscient écologique. L'affranchissement du symbolique, médiateur fondamental dans le rapport au monde, a permis l'établissement du dialogue fondateur. (Cottereau, 2001, p. 135)

Lors de cette phase, le projet créatif se définit plus clairement ; on observe une mise en tion intuitive. Les paroles laissent place à un bourdonnement d'actions silencieuses, cette sorte de bulle qui se crée, comme dans le cas du dessin, c'est le mode de perception créatif du cerveau qui est mis à profit, avec une relation particulière au dans lequel s'inscrit le geste qui prend sens. La projection dans l'avenir ou dans le passé n'a pas d'importance.

une grande unité physique à laquelle nous sommes liés. Lentement, dans cette mise en action du corps et de l'esprit, un sentiment de bien-être apparaît, l'impression intense niveau. L'aliénation fait place à l'engagement, l'enchantement remplace l'ennui » (Mihaly Csikszentmihalyi, cité dans Terstegge, 2013, p. 27).

est donc à travers ce processus créatif immersif, à travers ce jeu de construction avec les lois naturelles en présence (gravité, plasticité et résistance des matériaux), que les 
participants retrouvent un peu de leur propre nature. Réalisée dans un esprit ludique et libre, mais avec tout le sérieux requis pour la production d'une œuvre d'art, cette activité éveille ou renouvelle un sentiment de confiance en soi qui peut persister bien après la randonnée. Dans cette action avec la matière naturelle brute, ces gestes, qui pourraient s'apparenter à ceux du cueilleur, du cultivateur ou du bâtisseur, réduisent peu à peu la distance qui nous sépare de la nature :

C'est en développant un dialogue particulier avec la nature, interactif et enraciné dans la réalité de l'expérience inscrite dans un lieu et dans un temps précis, et en donnant à ces éléments une voix égale dans le processus créateur, qu'au bout du compte, nous respectons le sentiment que nous avons de faire partie intégrante de l'environnement. (Grande, 1997, p. 50)

Cette approche, qui propose une action artistique en lien avec les éléments, s'inscrit dans le courant holistique de l'ERE, lequel vise à « développer les multiples dimensions de son être en interaction avec les multiples dimensions de l'environnement » (Sauvé, 1997, p. 156) et plus particulièrement, aux pratiques que propose le courant de l'écopédagogie.

\section{Phase de séparation}

Lorsque l'œuvre est terminée, la plupart des participants éprouvent un sentiment de satisfaction, celui d'avoir accompli un travail qui a une signification pour eux. Ce sentiment est habituellement accompagné d'un vif besoin de partager avec les autres. Les participants sont alors invités à faire ensemble le tour des œuvres. Les créateurs comme les observateurs commentent chacune des œuvres et partagent leurs expériences. Le jugement n'est pas présent dans cette forme d'art. C'est au moment de la verbalisation que toute la dimension symbolique des formes créées et toute la profondeur personnelle du travail apparaissent. Au-delà des amoncellements physiques de pierres, de bâtons et de feuilles, c'est souvent du rapport à la vie, à la mort, au temps, de son propre rapport à la nature dont il est question. Cette phase implique une séparation de l'œuvre éphémère dont on ne gardera qu'une trace photographique. Ce détachement et le caractère éphémère de l'œuvre renvoient le participant à l'acceptation positive de sa propre existence éphémère qui s'inscrit dans le grand cycle naturel de la vie.

Les participants ressortent généralement de cette expérience avec le sentiment d'avoir l'âme nourrie, d'avoir véritablement vécu un moment privilégié. Un moment et des sensations qui restent gravés dans la mémoire avec un sentiment de confiance, d'apaisement et d'ouverture aux autres et au monde.

La figure 1 synthétise les caractéristiques des trois phases du processus que nous venons de décrire. 


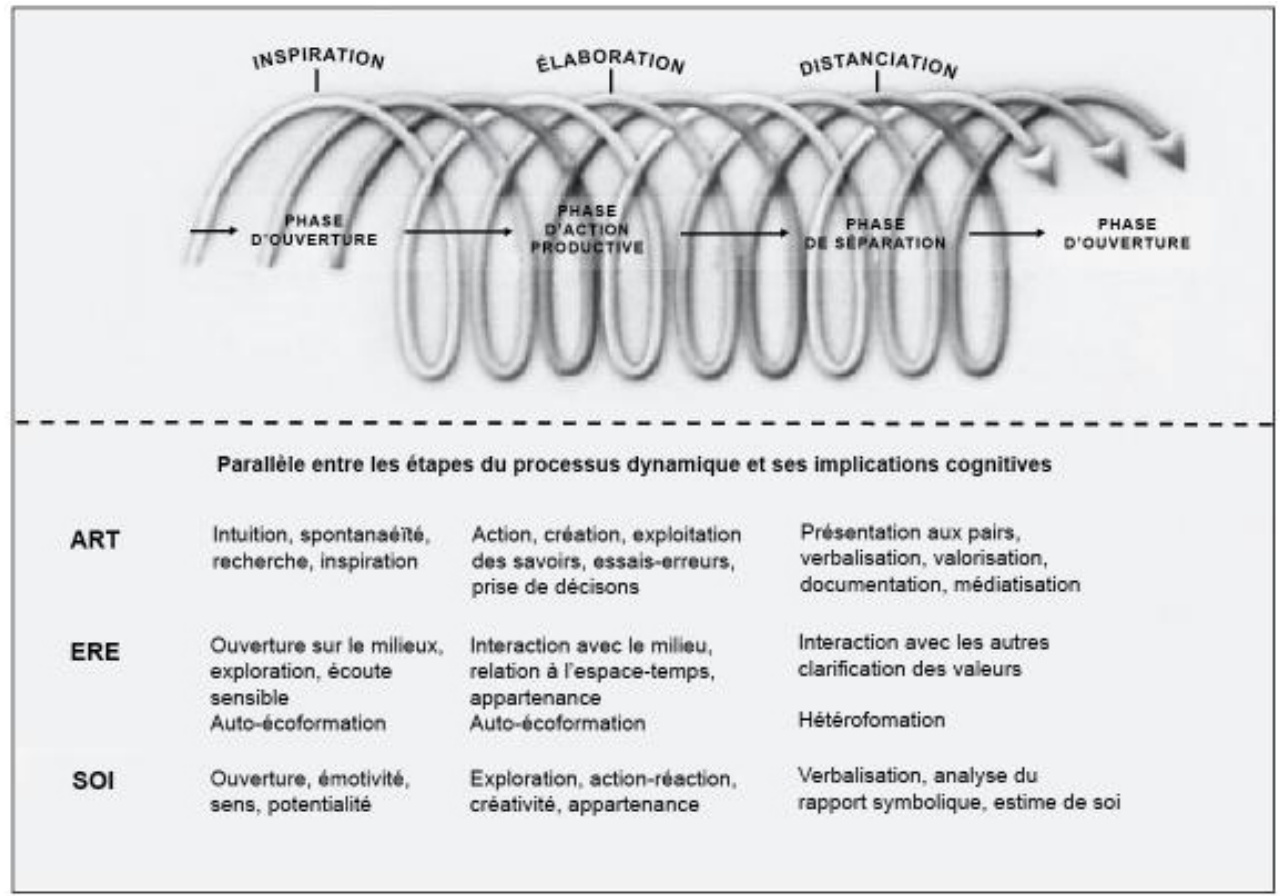

Figure 1 : Spécificités du processus dynamique de création d'art nature (d'après La dynamique de création de Gosselin, Potvin, Gingras et Murphy, 1998)

\section{Conclusion}

Le mont Royal, par ses richesses, par sa grande valeur symbolique et identitaire, semble demeurer une priorité en matière de préservation dans le cœur des élus et dans celui de la population. Une vigilance constante y reste cependant toujours nécessaire, car rien n'est jamais acquis.

En effet, en ce début de siècle, les questions d'environnement et de solidarité, qui constituent les fondements mêmes de la vie humaine, ne semblent plus faire le poids dans la balance décisionnelle face à l'économie néolibérale mondialisée. Cette économie, loin d'être une science exacte répondant à une loi naturelle invisible comme on aime nous la présenter, semble bien souvent répondre à des critères d'ordre subjectif et s'appuie sur le partage de valeurs plus émotives que rationnelles, à commencer par celle de l'argent : «La performance économique conditionne nos vies ; elle nous obsède et est devenue la seule réalité au point de nous faire négliger la qualité de notre environnement et de notre bien-être. L'être humain du $21^{\mathrm{e}}$ siècle est schizophrène » (Lefèvre, 2016, p. 81).

Dans un tel contexte, l'ERE doit maintenant intégrer à ses visées et ses pratiques la dimension symbolique et affective du rapport à l'environnement, à soi et aux autres. Ce faisant, elle pourra mieux contribuer à rétablir dans l'échelle décisionnelle, les valeurs fondamentales qui sont propres à la vie.

39 À l'appui de plusieurs chercheurs (Cotereau, 2001 ; Louv, 2008; Sobel, 1996), nous adoptons le postulat que la multiplication des contacts sensibles avec la nature, notamment à travers le processus créatif, contribuera au rétablissement d'un rapport sain et harmonieux à soi-même et à l'environnement. L'art nous propose une approche 
dynamique, existentielle, holistique et positive, qui restaure la confiance en soi et amène à revoir le fondement de nos valeurs. Une telle confiance pourrait servir d'assise à un engagement citoyen au cœur de la «cité » d'un monde partagé. Mon père, qui a consacré sa vie à la pratique et l'enseignement de l'agrobiologie, disait : « pour nous, la terre est une responsabilité heureuse. L'écologie n'est pas une chose triste $»^{5}$. L'art associé à l'ERE peut constituer un formidable moyen de contribuer à cette nécessaire et surtout heureuse transition écologique pour nos sociétés. Là où la science et la conscience semblent échouer, l'espoir nous mènerait à croire, comme le disait Fiodor Dostoïevski, que « l'art sauvera le monde».

\section{BIBLIOGRAPHIE}

Chartier, D. (2008). La vision de Frederick Law Olmsted relative au mont Royal. Directions des grands parcs et de la nature en ville. Ville de Montréal.

Cottereau, D. (2001). Formation entre terre et mer. Alternance écoformatrice. Condé-surNoireau : Éditions L'Harmattan.

Edwards, B. (1979). Dessiner grâce au cerveau droit. Liège : Pierre Mardaga Éditeur.

Gosselin, P., Potvin, G., Gingras, J-M. et Murphy, S. (1998). Une représentation de la dynamique de création pour le renouvellement des pratiques en éducation artistique. Revue des sciences de l'éducation, vol. XXIV, no 3, p. 647-666.

Grande, K. J. (1997). Art, nature et société. Montréal : Éditions Écosociété.

Lefèvre, T. (2016). Sortir de l'impasse. Qu'est-ce qui freine la transition écologique ? Paris :

Éditions Multimonde.

Loncol Daigneault, L. (2011). Art et nature au Québec : les trajectoires et les résidences de Boréal art/nature (mémoire de maîtrise), Université du Québec À Montréal.

Louv, R. (2008). Last child in the woods : Saving our children from nature-deficit disorder. Chapel Hill, N.C. : Algonquin Books.

Kastner, J. (2005). Land art et art environnemental. Londres : Édition Phaïdon, Collection «Themes \& Movements ».

Pineau, G. (1977). Éducation ou aliénation permanente ? Paris : Éditions Sciences et culture Montréal.

Sauvé, L. (1997). Pour une éducation relative à l'environnement. Montréal : Guérin Éditeur.

Sobel, D. (1996). Beyond ecophobia. Great Barrington, MA : Orion Society.

Terstegge, M. (2013). Le flow : vivez les bienfaits de l'expérience optimale. Montréal : Les Éditions de l'Homme.

Vidal, C et Benoit-Browaeys, D. (2005). Cerveau - Sexe et pouvoir. Paris : Éditions Belin. 


\section{NOTES}

1. Au Québec, ces symposiums proposent, ou ont proposé, à de nombreux artistes professionnels et amateurs provenant de toute la province et parfois du monde entier, des espaces de création en interaction avec le milieu naturel, notamment : Boréal Art Nature, programme de résidences, Rivière rouge; Création sur le champ / Land art Mont-Saint-Hilaire, Mont-Saint-Hillaire; Festival international de jardins, Jardin de Métis, Grand-Métis; Symposium international d'art-nature de Val-David, Jardin du précambrien, Val David (1996 à 2015); Symposium Cime et Racines, Site du Parc la Gabelle, Mauricie (2000 et 2001); Symposium art-nature, Pierre qui roule, Saint-AndréAvellin. (2006 à 2015). Parmi les artistes québécois les plus connus qui participent à ces évènements et à cette mouvance, notons René Derouin, Sonia Robertson, Armand Vaillancourt et Bill Vazan.

2. L'art nature (ou land art en anglais) est un courant d'art contemporain qui est apparu dans les années soixante aux États-Unis. À l'origine, les artistes, tels que Robert Smithson ou Walter de Maria, souhaitaient quitter les musées pour créer des œuvres in situ proposant au public une expérience signifiante avec l'environnement extérieur. Au début de ce mouvement artistique, les œuvres gigantesques sont créées avec l'utilisation d'engins mécaniques et modifient durablement le paysage. Très vite cependant, un courant naturaliste émerge avec la volonté d'établir dans son processus créatif un rapport plus harmonieux avec le milieu naturel. L'art nature tend à se distancier de l'art contemporain conceptuel qui s'adresse surtout à un public d'initiés. Plusieurs artistes tels que Nils Udo et Andy Goldsworthy n'utilisent comme outils que leurs mains et les éléments naturels trouvés sur place pour créer des œuvres éphémères intégrées au milieu naturel. Cette forme d'art accessible tant dans sa compréhension que dans sa pratique est actuellement privilégiée par de nombreux pédagogues pour son riche potentiel en éducation relative à l'environnement.

3. Pour plus d'information sur l'organisme et sa programmation : www.lemontroyal.qc.ca

4. Organisme à but non lucratif basé à Montréal, Les amis de la montagne a pour mission de protéger et mettre en valeur le mont Royal en privilégiant l'engagement de la communauté, l'éducation relative à l'environnement et la représentation des intérêts de la montagne afin de favoriser la prise de décisions éclairées en appui à la pérennité du lieu. Le service éducatif de l'organisme propose notamment une série d'actions et de programmes éducatifs pour les publics corporatifs ou scolaires et pour le grand public.

5. Article paru le 16 janvier 2016, Journal Midi libre, « Yvon Montigné, le père de l'agriculture biologique nous a quittés. "

\section{RÉSUMÉS}

Les Amis de la montagne est un organisme de protection et de mise en valeur du mont Royal qui propose depuis quelques années, au grand public et aux groupes de jeunes, des randonnées de création art nature en forêt. Au cours de ces randonnées, les participants sont invités à dessiner l'environnement, puis à créer par la suite des œuvres d'art in situ à partir des éléments naturels. L'art sert ici de vecteur pour un lien sensible au milieu naturel. La dynamique spécifique du processus créatif implique un mode de perception différent de notre rapport au temps et à l'espace. Ce processus favorise une connexion plus harmonieuse à soi, au milieu et aux autres. Ce type d'approche - sensible, symbolique, holistique, voire existentielle - pourrait davantage être 
mis à profit dans les pratiques d'éducation relative à l'environnement pour contribuer à la réflexion et à la restructuration de nos valeurs fondamentales vers un bien vivre ensemble.

For the past few years, Les amis de la montagne, whose mission is to protect and enhance Mount Royal, has offered nature art walks for the public and youth groups. During the walks, participants spend time drawing the surroundings and creating artworks using natural materials. Art puts them in touch with nature. The specific dynamics of the creative process involve changes in the way we perceive time and space. The process helps us reconnect with the environment, others and ourselves. This type of approach - sensitive, symbolic, holistic, even existential - could be used in educational activities about the environment to help restore our fundamental values and facilitate the transition to a sustainable future.

\section{INDEX}

Index géographique : Québec

Mots-clés : art, dessin, peinture, land art, approche sensible, art nature, éducation relative à l'environnement, Mont Royal

Keywords : art, drawing, paint, land art, nature art, sensitive approach, environmental education, Mount Royal

\section{AUTEUR}

\section{SAMUEL MONTIGNÉ}

Titulaire d'un baccalauréat en enseignement des arts visuels et d'un diplôme de second cycle en éducation relative à l'environnement de l'Université du Québec à Montréal, Samuel Montigné approfondit ses recherches sur les relations entre art, nature et ERE en adoptant une double posture de chercheur-pédagogue et d'artiste. Chargé de projets depuis plus de dix ans aux Amis de la montagne, il conçoit et conduit des programmes pédagogiques pour le grand public et pour le public scolaire ainsi que des évènements culturels et des projets d'expositions sur le mont Royal. 